\title{
Based on fuzzy comprehensive evaluation method of college students' sports life style and the physical quality correlation is explored
}

\author{
Xueliang Zhang \\ Department of P.E and Art Education, Zhejiang YueXiu University of Foreign Languages, Shaoxing 312000, China
}

\begin{abstract}
With people living standard rise, the teenagers' physical quality decline has become a universal phenomenon in China and the world, more and more people begin to pay close attention to health problems, for example, the old people's physical quality, the physical health of children, the physical health of teenagers. Teenagers as the backbone of the society, their physical condition is extra attention. In this paper, using principal component analysis (pca) on the influencing factors of health are principal component extraction, extraction of principal component for pull-ups in succession, crook proneness and lung capacity. From different way of life analysis of the physical quality of college students, a set of sports lifestyle including dimensions and dimensions of two groups, three groups of dimensionality and bad group. Through the fuzzy comprehensive evaluation method to carry on the analysis, the conclusion is physical quality best of three groups of dimensions, followed by the dimensions of two groups, the dimensions of three groups, the bad group. This conclusion conforms to the actual situation.
\end{abstract}

\section{Introduction}

Physical quality decline of teenagers has become a universal phenomenon in China and the world. Adolescents are an important force in the national development and national rejuvenation. Their physical quality influence the development of society in the future. Therefore, teenagers strengthen physical training, enhance physical quality, has become the focus of many experts [1-4].

In reference on the basis of a large number of literature, this paper will take the boy as the research object, considering various factors of judge boys physical model is established, in order to further study of this problem.

\section{Model establishment}

The purpose of the model establishment is establishing students physical quality evaluation relationships, utilizes students' physical quality relations to prove sorts ways rationality. Take Shenyang Normal University schoolboys as examples, targeted at sports way dimension one group, dimension two groups, dimension group three and defective group, it makes research. Firstly, utilize principal component analysis method to extract all influence factors, and then utilize fuzzy comprehensive evaluation method to evaluate the four groups, finally, analyze results [5-7].

\subsection{Data collection}

\footnotetext{
a Corresponding author: XueliangZhang66@163.com
}

Dimension refers to sports lifestyle, sports lifestyle totally has three types, own any one kind of them is called dimension one group, own any two groups of them is called dimension two groups, and own all the three kinds is called dimension three groups. Table 1-3 data is from the article 'University students' sports lifestyle and physique correlation research - take Shenyang Normal University as an example".

From Table 1, we can see dimension one group and defective group have significant differences in lung capacity, while other items differences are insignificant.

Table 1. Schoolboys sports ways dimension one group and defective group relations.

\begin{tabular}{ccc}
\hline & Dimension one & Defective group \\
\hline Pull-up(beats) & 5.78 & 5.32 \\
Sit and reach $(\mathrm{cm})$ & 7.5 & 6.45 \\
Lung capacity $(\mathrm{ml})$ & 3789.28 & 3756.67 \\
Height $(\mathrm{cm})$ & 172.87 & 174.43 \\
Weight $(\mathrm{kg})$ & 66.38 & 67.23 \\
Lung body index $(\mathrm{ml} / \mathrm{kg})$ & 58.48 & 57.64 \\
Grip $(\mathrm{kg})$ & 42.36 & 41.85 \\
Grip index & 64.51 & 63.75 \\
50m(sec) & 8.03 & 8.01 \\
Standing long jump(cm) & 217.72 & 220.17 \\
1000m(sec) & 271.27 & 280.09 \\
\hline
\end{tabular}

From Table 2 we can see dimension two groups and defective group except for having significant differences in lung capacity; they also have gaps in sit and reach item. 
Table 2. Schoolboys sports ways dimension two groups and defective group relations.

\begin{tabular}{ccc}
\hline & Dimension two & Defective group \\
\hline Pull-up (beats) & 5.74 & 5.32 \\
Sit and reach $(\mathrm{cm})$ & 8.03 & 6.45 \\
Lung capacity $(\mathrm{ml})$ & 3673.32 & 3756.67 \\
Height $(\mathrm{cm})$ & 173.09 & 174.43 \\
Weight $(\mathrm{kg})$ & 67.41 & 67.23 \\
Lung body index $(\mathrm{ml} / \mathrm{kg})$ & 55.57 & 57.64 \\
Grip $(\mathrm{kg})$ & 42.39 & 41.85 \\
Grip index & 63.47 & 63.75 \\
50m (sec) & 7.99 & 8.01 \\
Standing long jump $(\mathrm{cm})$ & 220.21 & 220.17 \\
1000m (sec) & 269.69 & 280.09 \\
\hline
\end{tabular}

Table 3. Schoolboys sports ways dimension three groups and defective group relations.

\begin{tabular}{ccc}
\hline & Dimension three & Defective group \\
\hline Pull-up (beats) & 6.4 & 5.32 \\
Sit and reach $(\mathrm{cm})$ & 8.42 & 6.45 \\
Lung capacity $(\mathrm{ml})$ & 3639.8 & 3756.67 \\
Height $(\mathrm{cm})$ & 175.02 & 174.43 \\
Weight $(\mathrm{kg})$ & 70.54 & 67.23 \\
Lung body index $(\mathrm{ml} / \mathrm{kg})$ & 52.49 & 57.64 \\
Grip $(\mathrm{kg})$ & 44.27 & 41.85 \\
Grip index & 64.24 & 63.75 \\
50m (sec) & 7.79 & 8.01 \\
Standing long jump (cm) & 227.14 & 220.17 \\
1000m (sec) & 256.95 & 280.09 \\
\hline
\end{tabular}

From Table 3 data, we can find that most of items have differences. Due to influence factors are numerous; therefore we adopt principal component analysis method to extract principal components.

\subsection{Principal component extraction}

Main way of principal component analysis is reducing dimension of variables, which is recombining original many variables with correlation into a group of uncorrelated variables to replace original variables [8]. Therefore, we can pay attention to every time observation's variables that have maximum variation, to every time observation's small changed variables that can be used as constant to process and get rid of them, so that it reduces variables number in problem that needs to be considered.

\subsection{Principal component theory}

Assume that there is $m$ pieces of original indicators to do principal component analysis, which are recorded as $x_{1}, x_{2}, \cdots, x_{m}$, now it has $n$ pieces of samples, corresponding observation value is $x_{i k}(i=1,2, \cdots, n)$, and $k=1,2, \cdots, m$ takes standardization transformation, and then transform $x_{k}$ into $x_{k}^{*}$, that: $x_{k}^{*}=\frac{x_{k}-\overline{x_{k}}}{s_{k}}, k=1,2, \cdots, m$.

Among them, $\overline{x_{k}}$ and $s_{k}$ are respectively $x_{k}$ average number and standard deviation, $x_{k}^{*}$ average number is 0 , standard deviation is 1 .

According to each sample original indicator observation value $x_{i k}$ or after standardization observation value $x_{i k}^{*}$, it solves coefficient $b_{k j}$, establish indicator $x_{k}^{*}$ that is transformed through standardization to express comprehensive indicator $z_{j}$ equation $z_{j}=\sum_{k} b_{k j} x_{k}^{*}$, which can also establish equation that uses original indicator $x_{k}$ to express comprehensive indicator $z_{j}: z_{j}=\sum_{k} \tilde{b}_{k j} x_{k}^{*}+a_{j}$. There are two requirements on defining $b_{k j}$ :

(1) Comprehensive indicators are mutual independent from each other or uncorrelated.

(2) Every comprehensive indicator reflected each sample gross information content is equal to corresponding feature vector (comprehensive indicator coefficient) feature values. In general, it is required that selected comprehensive indicator feature vales contribution ratios sum to be above $80 \%$.

\subsection{Principal component extraction result}

Combine with above theoretical process, utilize SPSS software, process with dimension one group, dimension two groups, dimension three groups and defective group data.

\subsection{Fuzzy comprehensive evaluation}

In general, fuzzy comprehensive evaluation involves three quantities. Set that there are $n$ pieces of evaluated objects correlation factors, it records as $U=\left\{u_{1}, u_{2}, \cdots, u_{n}\right\}$, and calls it as factor set. And set all possible occurred remarks have $m$ pieces, it records as $V=\left\{v_{1}, v_{2}, \cdots, v_{m}\right\}$, and calls it as evaluation set. Because every factor position is different, its function is also different, it appears measurement criterion that is weight, and it records $A=\left\{a_{1}, a_{2}, \cdots, a_{n}\right\}$.

\subsection{Comprehensive evaluation steps.}

Fuzzy comprehensive evaluation steps are proceeding as following methods:

(1)Define factor set $U=\left\{u_{1}, u_{2}, \cdots, u_{n}\right\}$.

(2)Define evaluation set $V=\left\{v_{1}, v_{2}, \cdots, v_{m}\right\}$.

(3)Carry out single factor evaluation and get $r_{i}=\left\{v_{i 1}, v_{i 2}, \cdots, v_{i m}\right\}$.

(4)Construct comprehensive evaluation matrix:

$R=\left[\begin{array}{cccc}r_{11} & r_{12} & \cdots & r_{1 m} \\ r_{21} & r_{22} & \cdots & r_{2 m} \\ \vdots & \vdots & & \vdots \\ r_{n 1} & r_{n 2} & \cdots & r_{n m}\end{array}\right]$ 
(5)Comprehensive evaluation: to weight

$A=\left\{a_{1}, a_{2}, \cdots, a_{n}\right\}$, calculate $B=A \circ R$, and need to make evaluation according to maximum membership principle.

\subsection{Operator definition}

When make comprehensive evaluation, according to operator different definitions, it has different models.

(1) Model I : $M(\wedge, \vee) —$ Principal divisor decisive type

Computing method is:

$$
b_{j}=\max \left\{\left(a_{i} \wedge r_{i j}\right), i=1,2, \cdots, n\right\}(j=1,2, \cdots, m)
$$

The model evaluation result is up to factors that play main effects on total evaluation, other factors will not impact on evaluation, relatively, the model is fit for the case that comprehensive evaluation is thought to be optimal when single evaluation is optimal.

(2) Model II : $M(\bullet, \vee) \longrightarrow$ Principal divisor prominent type

Computing method is:

$$
b_{j}=\max \left\{\left(a_{i} \bullet r_{i j}\right), i=1,2, \cdots, n\right\}(j=1,2, \cdots, m)
$$

The model has some similarities with I model, but it is more refining than I model. It not only highlights main factors, but also gives considerations to other factors. The model is fit for the range that model I is inapplicable, which is also in case that each factor cannot be distinguished but needed to be refined.

(3) Model III: $M(\bullet,+) \_$Weighted average type

Computing method is:

$$
b_{j}=\sum_{i=1}^{n} a_{i} \bullet r_{i j}(j=1,2, \cdots, m)
$$

The model according to each factor importance, take all influence factors into consideration, relatively it is fit for the case that requires comprehensive optimization.

(4)Model IV : $M(\wedge, \oplus)$ - Taking sum of small upper bound type

Computing method is:

$$
b_{j}=\min \left\{\left(1, \sum_{i=1}^{n}\left(a_{i} \wedge r_{i j}\right)\right)\right\}(j=1,2, \cdots, m)
$$

When use the model, it should pay special attention to: every $a_{i}$ cannot take excessive big value, otherwise it may appear the case that $b_{j}$ is 1 ; every $a_{i}$ cannot take excessive small value, otherwise it will appear the case that $b_{j}$ is equal to the sum of each $a_{i}$, which will lead to single factor evaluation relative information lose.

(5)Model V:M(^,+)—Balanced average type

Computing method is:

$$
b_{j}=\sum_{i=1}^{n}\left(a_{i} \wedge \frac{r_{i j}}{r_{0}}\right)(j=1,2, \cdots, m)
$$

Among them, $r_{0}=\sum_{k=1}^{n} r_{k j}$. The model is fit for comprehensive evaluation matrix $R$ element is excessive big or small cases.

The paper established model uses principal divisor decisive type's operator.

\subsection{Establish process}

The paper through dimension one, two, three group and defective group evaluation, establish a group of evaluating physical quality reasonable model, so as to later period relative problems research. By principal component analysis, we define three main factors they are respectively pull-up, sit and reach, lung capacity. Therefore, we need to establish the problem factor set $U=\left\{u_{1}, u_{2}, u_{3}\right\}$, from which $u_{1}$ represents pull-up, $u_{2}$ represents sit and reach, $u_{3}$ represents lung capacity. In order to make comparison among dimension one group, dimension two groups, dimension three groups and defective group data, we draw broken line graph as Figure 1 shows.

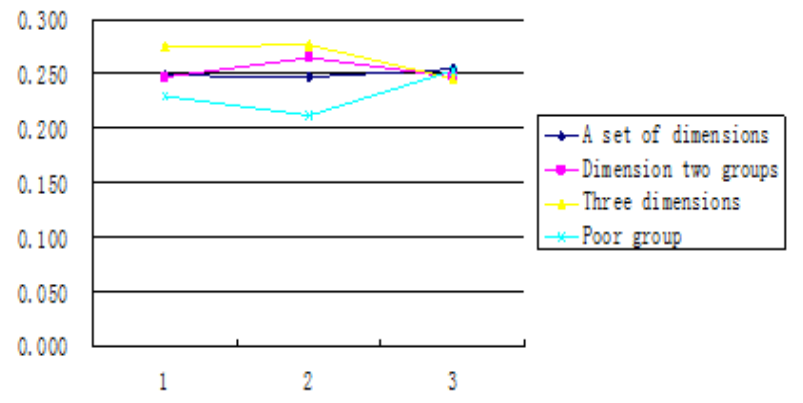

Figure 1. Comparison of the four groups.

From Figure 1, we can see that differences in pull-up and sit-and-reach two items are larger, while differences in lung capacity are relative small.

According to experience, we set lung capacity weight value as 0.4 , both sit and up and pull-up weight values as 0.3 . We can see the evaluation physical quality process used weight is: $A=(0.3,0.3,0.4)$

According to Table 5 data, establish fuzzy comprehensive evaluation matrix:

$$
R=\left[\begin{array}{llll}
0.249 & 0.247 & 0.275 & 0.229 \\
0.247 & 0.264 & 0.277 & 0.212 \\
0.255 & 0.247 & 0.245 & 0.253
\end{array}\right]
$$

Take model I $-M(\wedge, \vee)$ as evidence weight, calculate and get: $B=A \circ R=(0.255,0.264,0.277,0.253)$

Therefore, by evaluation, dimension one group evaluation result is 0.255 , dimension two groups evaluation result is 0.264 , dimension three groups evaluation result is 0.277 , defective group evaluation result is 0.253 , carry out standardized processing with evaluation result.

\section{Conclusion}


The paper used fuzzy comprehensive evaluation method generally will solve a kind of arrangement and selective difficulties. The key to whole process is establishing fuzzy comprehensive evaluation matrix. The method is easier to use, has smaller limitations, therefore, it is widely applied in all kinds of problems. But the method also has its drawbacks, used weights in the process, in general, it needs artificial estimation, once weight is unreasonable, it will lead to result irrationality. The paper applies fuzzy comprehensive evaluation into researching on university students' sports exercising ways and physical quality problems, obtained result are dimension three groups' physical quality is best, defective group physical quality is worst. The result conforms to practical situation. Established evaluation model conforms to practical life rules. Therefore, the model can popularize in other similar problems. Dimension three groups own sports three kinds of life habits, therefore, relative department should encourage students to form into comprehensive sports life habits.

\section{References}

1. Y.X. Yang, Journal of Anhui Sports Science. 34, 35-37 (2013).

2. D.M. Jahin, Journal of Mechanical Engineering Research and Developments. 39, 97-100 (2016).

3. L. Li, Z.Q. Mi, S.Y. Bi, Journal of Hubei Sports Science. 32, 510-511 (2013).

4. X. Hu, J.S. Zhou, H.F. Tan, R. Shen, Journal of Beijing Sport University. 27, 281-282 (2004).

5. X.L. Zhang, J. Wang, Journal of Guangzhou Physical Education Institute. 26, 67-69 (2006).

6. G.M. Kristina, C.E. Gooyers, Journal of Mechanical Engineering Research and Developments. 39, 204-210 (2016).

7. Y. Liu, J.B. Liu, Journal of Capital College of Physical Education. 19, 122-124 (2007).

8. Z.L. Liu, Biotechnology: An Indian Journal, 10, 1883-1886 (2014). 